\title{
Brauer Groups of Quot Schemes
}

\author{
Indranil Biswas, Ajneet Dhillon, \& Jacques Hurtubise
}

Abstract. Let $X$ be an irreducible smooth complex projective curve. Let $\mathcal{Q}(r, d)$ be the Quot scheme parameterizing all coherent subsheaves of $\mathcal{O}_{X}^{\oplus r}$ of rank $r$ and degree $-d$. There are natural morphisms $\mathcal{Q}(r, d) \longrightarrow \operatorname{Sym}^{d}(X)$ and $\operatorname{Sym}^{d}(X) \longrightarrow \operatorname{Pic}^{d}(X)$. We prove that both these morphisms induce isomorphism of Brauer groups if $d \geq 2$. Consequently, the Brauer group of $\mathcal{Q}(r, d)$ is identified with the Brauer group of $\operatorname{Pic}^{d}(X)$ if $d \geq 2$.

\section{Introduction}

Let $X$ be an irreducible smooth projective curve defined over $\mathbb{C}$. For any integer $r \geq 1$, consider the trivial holomorphic vector bundle $\mathcal{O}_{X}^{\oplus r}$ on $X$. For any $d \geq 0$, let $\mathcal{Q}(r, d)$ denote the Quot scheme that parameterizes all torsion quotients of degree $d$ of the $\mathcal{O}_{X}$-module $\mathcal{O}_{X}^{\oplus r}$. This $\mathcal{Q}(r, d)$ is an irreducible smooth complex projective variety of dimension $r d$.

For every $Q \in \mathcal{Q}(r, d)$, we have a corresponding short exact sequence

$$
0 \longrightarrow \mathcal{F}(Q) \stackrel{\rho}{\longrightarrow} \mathcal{O}_{X}^{\oplus r} \longrightarrow Q \longrightarrow 0 .
$$

The pairs $\left(\mathcal{O}_{X}^{\oplus r}\right)^{*}=\mathcal{O}_{X}^{\oplus r} \stackrel{\rho^{*}}{\longrightarrow} \mathcal{F}(Q)^{*}$ are vortices of a particular numerical type. The Quot scheme $\mathcal{Q}(r, d)$ is a moduli space of vortices of a particular numerical type (see [BDW; Ba; BR], and references therein).

Sending such $Q$ to the scheme theoretic support of the quotient for the homomorphism

$$
\bigwedge^{r} \mathcal{F}(Q) \longrightarrow \bigwedge^{r} \mathcal{O}_{X}^{\oplus r}
$$

induced by the inclusion $\mathcal{F}(Q) \longrightarrow \mathcal{O}_{X}^{\oplus r}$, we get a morphism

$$
\varphi: \mathcal{Q}(r, d) \longrightarrow \operatorname{Sym}^{d}(X) \text {. }
$$

Sending any $Q \in \mathcal{Q}(r, d)$ to the holomorphic line bundle $\wedge^{r} \mathcal{F}(Q)^{*}$, we get a morphism

$$
\varphi^{\prime}: \mathcal{Q}(r, d) \longrightarrow \mathcal{Q}(1, d)=\operatorname{Pic}^{d}(X) .
$$

On the other hand, we have the morphism

$$
\xi_{d}: \operatorname{Sym}^{d}(X) \longrightarrow \operatorname{Pic}^{d}(X)
$$

that sends any $\left(x_{1}, \ldots, x_{d}\right)$ to the holomorphic line bundle $\mathcal{O}_{X}\left(\sum_{i=1}^{d} x_{i}\right)$. Note that $\varphi^{\prime}=\xi_{d} \circ \varphi$.

Received May 22, 2014. Revision received April 15, 2015. 
The cohomological Brauer group of a smooth complex projective variety $M$ will be denoted by $\operatorname{Br}^{\prime}(M)$. A theorem of Gabber says that $\operatorname{Br}^{\prime}(M)$ coincides with the Brauer group of $M$ (see [dJ]).

Our aim here is to prove the following:

THEOREM 1.1. For the morphisms $\varphi$ and $\xi_{d}$, the pullback homomorphisms of Brauer groups

$$
\begin{aligned}
& \varphi *: \operatorname{Br}^{\prime}\left(\operatorname{Sym}^{d}(X)\right) \longrightarrow \operatorname{Br}^{\prime}(\mathcal{Q}(r, d)) \quad \text { and } \\
& \xi_{d}^{*}: \operatorname{Br}^{\prime}\left(\operatorname{Pic}^{d}(X)\right) \longrightarrow \operatorname{Br}^{\prime}\left(\operatorname{Sym}^{d}(X)\right)
\end{aligned}
$$

are isomorphisms if $d \geq 2$.

Theorem 1.1 is proved in Lemma 4.2 and Lemma 6.1.

If genus $(X)=1$, then $\operatorname{Sym}^{d}(X)$ is a projective bundle over $X$, and hence $\operatorname{Br}^{\prime}\left(\operatorname{Sym}^{d}(X)\right)=0$. If $\operatorname{genus}(X)=0$, then $\operatorname{Br}^{\prime}\left(\operatorname{Sym}^{d}(X)\right)=0$ because $\operatorname{Sym}^{d}(X)=\mathbb{C P}^{d}$. Therefore, Theorem 1.1 has the following corollary:

Corollary 1.2. If genus $(X) \leq 1$, then $\operatorname{Br}^{\prime}(\mathcal{Q}(r, d))=0$.

Since $\mathcal{Q}(r, 1)$ is a projective bundle over $X$, it follows that $\operatorname{Br}^{\prime}(\mathcal{Q}(r, d))=0$. Note that $\operatorname{Br}^{\prime}\left(\operatorname{Pic}^{d}(X)\right)$ is nonzero if genus $(X)>1$, whereas $\operatorname{Br}^{\prime}\left(\operatorname{Sym}^{1}(X)\right)=0$.

Fixing a point $x_{0} \in X$, construct an embedding

$$
\delta: \mathcal{Q}(r, d) \longrightarrow \mathcal{Q}(r, d+r)
$$

by sending any subsheaf $\mathcal{F} \subset \mathcal{O}_{X}^{\oplus r}$ to $\mathcal{F} \otimes \mathcal{O}_{X}\left(-x_{0}\right)$.

The following is proved in Corollary 6.2:

Proposition 1.3. The pullback homomorphism for $\delta$

$$
\delta^{*}: \operatorname{Br}^{\prime}(\mathcal{Q}(r, d+r)) \longrightarrow \operatorname{Br}^{\prime}(\mathcal{Q}(r, d))
$$

is an isomorphism if $d \geq 2$.

Now assume that $r$, genus $(X) \geq 2$; if $r=2$, then also assume that genus $(X) \geq 3$. Iterating the morphism $\delta$, we get an ind-scheme. This ind-scheme has the cohomology isomorphic to the moduli stack; see [Dh, Theorem 4.5] or [Ne, Chapter 4]. Using Proposition 1.3 and Theorem 1.1, we can now describe the cohomological Brauer group of the moduli stack of rank $r$ and degree $d$ bundles. Further, we deduce that the cohomological Brauer group of the moduli stack of vector bundles on $X$ of rank $r$ and fixed determinant vanishes. This result was proved earlier in [BH, Theorem 5.2]. Using this vanishing result, we can deduce that the cohomological Brauer group of the moduli space of stable vector bundles on $X$ of rank $r$ and fixed determinant of degree $d$ is a cyclic group of order g.c.d. $(r, d)$. This result was proved earlier in [BBGN]. 


\section{Cohomological Brauer Group}

Let $M$ be an irreducible smooth projective variety defined over $\mathbb{C}$. Let $\mathcal{O}_{M}^{*}$ denote the multiplicative sheaf on $M$ of holomorphic functions with values in $\mathbb{C} \backslash\{0\}$. The cohomological Brauer group $\operatorname{Br}^{\prime}(M)$ is the torsion subgroup of the cohomology group $H^{2}\left(M, \mathcal{O}_{M}^{*}\right)$.

Let $\mathcal{O}_{M}$ denote the sheaf of holomorphic functions on $M$. Consider the short exact sequence of sheaves on $M$

$$
0 \longrightarrow \mathbb{Z} \longrightarrow \mathcal{O}_{M} \stackrel{\exp }{\longrightarrow} \mathcal{O}_{M}^{*} \longrightarrow 0,
$$

where the homomorphism $\mathbb{Z} \longrightarrow \mathcal{O}_{M}$ sends any integer $n$ to the constant function $2 \pi \sqrt{-1} \cdot n$. Let

$$
\operatorname{Pic}(M)=H^{1}\left(M, \mathcal{O}_{M}^{*}\right) \stackrel{c}{\longrightarrow} H^{2}(M, \mathbb{Z}) \longrightarrow H^{2}\left(M, \mathcal{O}_{M}\right)
$$

be the corresponding long exact sequence of cohomology groups. The homomorphism $c$ in (2.1) sends a holomorphic line bundle to its first Chern class. The image $c(\operatorname{Pic}(M))$ coincides with the Néron-Severi group

$$
\mathrm{NS}(M):=H^{1,1}(M) \cap H^{2}(M, \mathbb{Z}) .
$$

Define the subgroup

$$
A:=H^{2}(M, \mathbb{Z}) / c(\operatorname{Pic}(M))=H^{2}(M, \mathbb{Z}) / \mathrm{NS}(M) \subset H^{2}\left(M, \mathcal{O}_{M}\right)
$$

(see (2.1)). Let

$$
H^{3}(M, \mathbb{Z})_{\text {tor }} \subset H^{3}(M, \mathbb{Z})
$$

be the torsion part.

Proposition 2.1 [Sco]. There is a natural short exact sequence

$$
0 \longrightarrow A \otimes_{\mathbb{Z}}(\mathbb{Q} / \mathbb{Z}) \longrightarrow \operatorname{Br}^{\prime}(M) \longrightarrow H^{3}(M, \mathbb{Z})_{\text {tor }} \longrightarrow 0,
$$

where $A$ is defined in (2.2).

See [Sco, p. 878, Proposition 1.1] for a proof of Proposition 2.1.

\section{The Cohomology of Symmetric Products}

Let $X$ be an irreducible smooth complex projective curve. The genus of $X$ will be denoted by $g$. For any positive integer $d$, let $P_{d}$ be the group of all permutations of $\{1, \ldots, d\}$. By $\operatorname{Sym}^{d}(X)$ we will denote the quotient of $X^{d}$ for the natural action

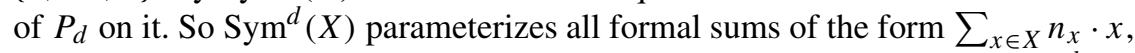
where $n_{x}$ are nonnegative integers with $\sum_{x \in X} n_{x}=d$. In other words, $\operatorname{Sym}^{d}(X)$ parameterizes all effective divisors on $X$ of degree $d$. This $\operatorname{Sym}^{d}(X)$ is an irreducible smooth complex projective variety of complex dimension $d$. Let

$$
q_{d}: X^{d} \longrightarrow \operatorname{Sym}^{d}(X)=X^{d} / P_{d}
$$

be the quotient map.

Let $\alpha_{1}, \alpha_{2}, \ldots, \alpha_{2 g}$ be a symplectic basis for $H^{1}(X, \mathbb{Z})$ chosen so that $\alpha_{i}$. $\alpha_{i+g}=1$ for $i \leq g$ and $\alpha_{i} \cdot \alpha_{j}=0$ if $|i-j| \neq g$. The oriented generator of 
$H^{2}(X, \mathbb{Z})$ will be denoted by $\omega$. For $i \in[1,2 g]$ and $j \in[1, d]$, we have the cohomology classes

$$
\lambda_{i}^{j}:=1 \otimes \cdots \otimes \alpha_{i} \otimes \cdots \otimes 1 \in H^{1}\left(X^{n}, \mathbb{Z}\right)
$$

and

$$
\eta^{j}:=1 \otimes \cdots \otimes \omega \otimes \cdots \otimes 1 \in H^{2}\left(X^{n}, \mathbb{Z}\right),
$$

where both $\alpha_{i}$ and $\omega$ are at the $j$ th position.

THEOREM 3.1 [Ma]. For the morphism $q_{d}$ in (3.1), the pullback homomorphism

$$
q_{d}^{*}: H^{*}\left(\operatorname{Sym}^{d}(X), \mathbb{Z}\right) \longrightarrow H^{*}\left(X^{d}, \mathbb{Z}\right)
$$

is injective. Further, the image of $q_{d}^{*}$ is generated, as a $\mathbb{Z}$-algebra, by

$$
\lambda_{i}=\sum_{j=1}^{d} \lambda_{i}^{j}, \quad 1 \leq i \leq 2 g, \quad \text { and } \quad \eta=\sum_{j=1}^{d} \eta^{j} .
$$

See [Ma, p. 325, (6.3)] and [Ma, p. 326, (7.1)] for Theorem 3.1.

There is a universal divisor $D^{\text {univ }}$ on $\operatorname{Sym}^{d}(X) \times X$, which consists of all $(z, x) \in \operatorname{Sym}^{d}(X) \times X$ such that $x$ is in the support of $z$. We wish to describe the class of this divisor in $H^{2}\left(\operatorname{Sym}^{d}(X) \times X, \mathbb{Z}\right)$. In view of the first part of Theorem 3.1, the algebra $H^{*}\left(\operatorname{Sym}^{d}(X) \times X, \mathbb{Z}\right)$ is considered as a subalgebra of $H^{*}\left(X^{d+1}, \mathbb{Z}\right)$.

For $i \in[1, d+1]$, let $\pi_{i}: X^{d+1} \longrightarrow X$ be the projection to the $i$ th factor. For any integer $k \in[1, d]$, consider the closed immersion

$$
\iota_{k}: X^{d} \hookrightarrow X^{d+1} m
$$

which is uniquely determined by

$$
\pi_{i} \circ \iota_{k}= \begin{cases}\pi_{i}^{\prime} & \text { if } i \neq d+1, \\ \pi_{k}^{\prime} & \text { if } i=d+1,\end{cases}
$$

where $\pi_{j}^{\prime}$ is the projection of $X^{d}$ to the $j$ th factor. In other words, $i_{k}\left(x_{1}, \ldots, x_{k}\right.$, $\left.\ldots, x_{d}\right)=i_{k}\left(x_{1}, \ldots, x_{k}, \ldots, x_{d}, x_{k}\right)$. The divisor on $X^{d+1}$ given by the image of $\iota_{k}$ will be denoted by $D_{k}$.

The divisor $D_{k}$ is closely related to the universal divisor $D^{\text {univ }}$ defined before. To see this, consider the projection

$$
q_{d} \times \operatorname{Id}_{X}: X^{d+1}=X^{d} \times X^{d} \longrightarrow \operatorname{Sym}^{d}(X) \times X,
$$

where $q_{d}$ is constructed in (3.1). The image $\left(q_{d} \times \operatorname{Id}_{X}\right)\left(D_{k}\right)$ is independent of the choice of $k$ and coincides with $D^{\text {univ }}$. This implies that $D^{\text {univ }}$ is irreducible.

The classes

$$
\lambda_{i}^{j} \cup \lambda_{i^{\prime}}^{j^{\prime}}, \quad i \neq i^{\prime}, 1 \leq j<j^{\prime} \leq d+1,
$$

and

$$
\eta^{j}, \quad 1 \leq j \leq d+1,
$$


constructed as in (3.2) and (3.3) for $d+1$, together give a basis for $H^{2}\left(X^{d+1}, \mathbb{Z}\right)$. We have the dual basis for $H^{2 d}\left(X^{d+1}, \mathbb{Z}\right)$ given by

$$
\eta^{j \vee}=\omega \otimes \cdots \otimes \omega \otimes 1 \otimes \omega \otimes \cdots \otimes \omega
$$

and

$$
\left(\lambda_{i}^{j} \cup \lambda_{i^{\prime}}^{j^{\prime}}\right)^{\vee}=\omega \otimes \cdots \otimes \omega \otimes \widetilde{\alpha}_{i} \otimes \omega \otimes \cdots \omega \otimes \widetilde{\alpha}_{i^{\prime}} \otimes \omega \otimes \cdots \otimes \omega,
$$

where $\widetilde{\alpha}_{i}$ (respectively, $\widetilde{\alpha}_{i^{\prime}}$ ) is the class with $\widetilde{\alpha}_{i} \cup \alpha_{i}=\omega$ (respectively, $\widetilde{\alpha}_{i^{\prime}} \cup \alpha_{i^{\prime}}=$ $\omega)$. Now

$$
\int_{D_{k}} \eta^{j \vee}=\int_{X^{d}} \iota_{k}^{*} \eta^{j \vee}= \begin{cases}1, & j=k, \\ 1, & j=d+1, \\ 0 & \text { otherwise },\end{cases}
$$

whereas

$$
\int_{D_{k}}\left(\lambda_{i}^{j} \cup \lambda_{i^{\prime}}^{j^{\prime}}\right)^{\vee}=\int_{X} \widetilde{\alpha}_{i} \cup \widetilde{\alpha}_{i^{\prime}}
$$

if $j^{\prime}=d+1$ and $j=k$, and

$$
\int_{D_{k}}\left(\lambda_{i}^{j} \cup \lambda_{i^{\prime}}^{j^{\prime}}\right)^{\vee}=0
$$

otherwise. So the class of $D_{k}$ is

$$
\eta^{k}+\eta^{d+1}+\sum_{i=1}^{g} \lambda_{i}^{k} \cup \lambda_{i+g}^{d+1}-\sum_{i=g+1}^{2 g} \lambda_{i}^{k} \cup \lambda_{i-g}^{d+1} .
$$

By the Künneth formula we have

$$
\begin{aligned}
& H^{2}\left(\operatorname{Sym}^{d}(X) \times X, \mathbb{Z}\right) \\
& \cong\left(H^{2}\left(\operatorname{Sym}^{d}(X), \mathbb{Z}\right) \otimes H^{0}(X, \mathbb{Z})\right) \\
& \quad \oplus\left(H^{0}\left(\operatorname{Sym}^{d}(X), \mathbb{Z}\right) \otimes H^{2}(X, \mathbb{Z})\right) \oplus\left(H^{1}\left(\operatorname{Sym}^{d}(X), \mathbb{Z}\right) \otimes H^{1}(X, \mathbb{Z})\right) .
\end{aligned}
$$

Using Theorem $3.1(3.1)$, we have a basis for $H^{2}\left(\operatorname{Sym}^{d}(X) \times X, \mathbb{Z}\right)$ consisting of $\eta \otimes 1_{X}, \quad\left\{\left(\lambda_{i} \cup \lambda_{j}\right) \otimes 1_{X}\right\}_{i, j=1}^{2 g}, \quad 1_{\operatorname{Sym}^{d}(X)} \otimes \omega, \quad\left\{\lambda_{i} \otimes \alpha_{j}\right\}_{i, j=1}^{2 g}$.

From the previous computations it follows that the class of $D^{\text {univ }}$ is

$$
\left[D^{\mathrm{univ}}\right]=\eta \otimes 1+d\left(1_{\operatorname{Sym}^{d}(X)} \otimes \omega\right)+\sum_{i=1}^{g} \lambda_{i} \otimes \alpha_{i+g}-\sum_{i=g+1}^{2 g} \lambda_{i} \otimes \alpha_{i-g} .
$$

Proposition 3.2.

(1) For a fixed point $x_{0} \in X$, consider the inclusion

$$
\iota_{x_{0}}: \operatorname{Sym}^{d}(X) \hookrightarrow \operatorname{Sym}^{d}(X) \times X
$$

defined by $z \longmapsto\left(z, x_{0}\right)$. The cohomology class $\iota_{x_{0}}^{*}\left[D^{\text {univ }}\right]$ is $\eta$.

(2) The slant product of $\left[D^{\mathrm{univ}}\right]$ with $\alpha_{i}^{\vee}$ produces the class $\lambda_{i}$ in $H^{1}\left(\operatorname{Sym}^{d}(X), \mathbb{Z}\right)$. 
Proof. These follow from (3.4).

\section{Cohomological Brauer Group of the Symmetric Product}

Recall that $X$ denotes a smooth projective curve. Fix a point $x_{0} \in X$. For any $d \geq 1$, let

$$
f_{d}: \operatorname{Sym}^{d}(X) \longrightarrow \operatorname{Sym}^{d+1}(X)
$$

be the morphism defined by $\sum_{x \in X} n_{x} \cdot x \longmapsto x_{0}+\sum_{x \in X} n_{x} \cdot x$. Let

$$
f_{d}^{*}: \operatorname{Br}^{\prime}\left(\operatorname{Sym}^{d+1}(X)\right) \longrightarrow \operatorname{Br}^{\prime}\left(\operatorname{Sym}^{d}(X)\right)
$$

be the pullback homomorphism for $f_{d}$ in (4.1).

LEMma 4.1. For every $d \geq 2$, the homomorphism $f_{d}^{*}$ in (4.2) is an isomorphism.

Proof. For every positive integer $d$, the cohomology group $H^{*}\left(\operatorname{Sym}^{d}(X), \mathbb{Z}\right)$ is torsionfree by Theorem 3.1. Therefore, from Proposition 2.1 we conclude that

$$
\operatorname{Br}^{\prime}\left(\operatorname{Sym}^{d}(X)\right) \cong\left(H^{2}\left(\operatorname{Sym}^{d}(X), \mathbb{Z}\right) / \operatorname{NS}\left(\operatorname{Sym}^{d}(X)\right)\right) \otimes_{\mathbb{Z}}(\mathbb{Q} / \mathbb{Z}) .
$$

From Theorem 3.1,

$$
H^{2}\left(\operatorname{Sym}^{d}(X), \mathbb{Z}\right)=\left(\bigwedge^{2} H^{1}(X, \mathbb{Z})\right) \oplus H^{2}(X, \mathbb{Z}) .
$$

Let

$$
f_{d}^{\prime}: H^{2}\left(\operatorname{Sym}^{d+1}(X), \mathbb{Z}\right) \longrightarrow H^{2}\left(\operatorname{Sym}^{d}(X), \mathbb{Z}\right)
$$

be the homomorphism that sends a cohomology class to its pullback by the map $f_{d}$ in (4.1). It is evident that in terms of the isomorphism in (4.4), this homomorphism $f_{d}^{\prime}$ coincides with the identity map of $\left(\bigwedge^{2} H^{1}(X, \mathbb{Z})\right) \oplus H^{2}(X, \mathbb{Z})$.

The isomorphism in (4.4) is clearly compatible with the Hodge decompositions. Since $f_{d}^{\prime}$ coincides with the identity map of $\left(\bigwedge^{2} H^{1}(X, \mathbb{Z})\right) \oplus H^{2}(X, \mathbb{Z})$, we now conclude that $f_{d}^{\prime}$ takes $\operatorname{NS}\left(\operatorname{Sym}^{d+1}(X)\right)$ isomorphically to $\operatorname{NS}\left(\operatorname{Sym}^{d}(X)\right)$. Therefore, the lemma follows from (4.3).

For any positive integer $d$, let

$$
\xi_{d}: \operatorname{Sym}^{d}(X) \longrightarrow \operatorname{Pic}^{d}(X)
$$

be the morphism defined by $\sum_{x \in X} n_{x} \cdot x \longmapsto \mathcal{O}_{X}\left(\sum_{x \in X} n_{x} \cdot x\right)$. Let

$$
\xi_{d}^{*}: \operatorname{Br}^{\prime}\left(\operatorname{Pic}^{d}(X)\right) \longrightarrow \operatorname{Br}^{\prime}\left(\operatorname{Sym}^{d}(X)\right)
$$

be the pullback homomorphism corresponding to $\xi_{d}$.

Lemma 4.2. For any $d \geq 2$, the homomorphism $\xi_{d}^{*}$ in (4.6) is an isomorphism.

Proof. Let

$$
\eta_{d}: \operatorname{Pic}^{d}(X) \longrightarrow \operatorname{Pic}^{d+1}(X)
$$


be the isomorphism defined by $L \longmapsto L \otimes \mathcal{O}_{X}\left(x_{0}\right)$. We have the commutative diagram

$$
\begin{array}{ccc}
\operatorname{Sym}^{d}(X) & \stackrel{f_{d}}{\longrightarrow} & \operatorname{Sym}^{d+1}(X) \\
\downarrow \xi_{d} & & \downarrow \xi_{d+1} \\
\operatorname{Pic}^{d}(X) & \stackrel{\eta_{d}}{\longrightarrow} & \operatorname{Pic}^{d+1}(X)
\end{array}
$$

where $f_{d}$ and $\xi_{d}$ are constructed in (4.1) and (4.5), respectively, and $\eta_{d}$ is defined above. Let



be the corresponding commutative diagram of homomorphisms of cohomological Brauer groups. From Lemma 4.1 we know that $f_{d}^{*}$ is an isomorphism for $d \geq 2$. The homomorphism $\eta_{d}^{*}$ is an isomorphism because the map $\eta_{d}$ is an isomorphism. Therefore, from the commutativity of (4.7) we conclude that the homomorphism $\xi_{d}^{*}$ is an isomorphism if $\xi_{d+1}^{*}$ is an isomorphism. Consequently, it suffices to prove the lemma for all $d$ sufficiently large.

As before, the genus of $X$ is denoted by $g$. Take any $d>2 g$. Note that for any line bundle $L$ on $X$ of degree $d$, using Serre duality, we have

$$
H^{1}(X, L)=H^{0}\left(X, K_{X} \otimes L^{\vee}\right)^{\vee}=0
$$

because degree $\left(K_{X} \otimes L^{\vee}\right)=2 g-2-d<0$.

Take a Poincaré line bundle $\mathcal{L} \longrightarrow X \times \operatorname{Pic}^{d}(X)$. From (4.8) it follows that the direct image

$$
\mathrm{pr}_{*} \mathcal{L} \longrightarrow \operatorname{Pic}^{d}(X)
$$

is locally free of rank $d-g+1$, where pr is the natural projection of $X \times \operatorname{Pic}^{d}(X)$ to $\operatorname{Pic}^{d}(X)$. The projective bundle $\mathbb{P}\left(\operatorname{pr}_{*} \mathcal{L}\right)$, that parameterizes the lines in the fibers of the holomorphic vector bundle $\mathrm{pr}_{*} \mathcal{L}$, is independent of the choice of the Poincaré line bundle $\mathcal{L}$. Indeed, this follows from the fact that any two choices of the Poincaré line bundle over $X \times \operatorname{Pic}^{d}(X)$ differ by tensoring with a line bundle pulled back from $\operatorname{Pic}^{d}(X)$ [ACGH, p. 166]. The total space of $\mathbb{P}\left(\mathrm{pr}_{*} \mathcal{L}\right)$ is identified with $\operatorname{Sym}^{d}(X)$ by sending a section to the divisor on $X$ given by the section; see [Scb]. This identification between $\operatorname{Sym}^{d}(X)$ and $\mathbb{P}\left(\mathrm{pr}_{*} \mathcal{L}\right)$ takes the map $\xi_{d}$ in (4.5) to the natural projection of $\mathbb{P}\left(\mathrm{pr}_{*} \mathcal{L}\right)$ to $\operatorname{Pic}^{d}(X)$.

Since $\mathbb{P}\left(\mathrm{pr}_{*} \mathcal{L}\right)$ is the projectivization of a vector bundle, the natural projection

$$
\mathbb{P}\left(\mathrm{pr}_{*} \mathcal{L}\right) \longrightarrow \operatorname{Pic}^{d}(X)
$$

induces an isomorphism of cohomological Bauer groups [Ga, p. 193]. Consequently, the homomorphism

$$
\xi_{d}^{*}: \operatorname{Br}^{\prime}\left(\operatorname{Pic}^{d}(X)\right) \longrightarrow \operatorname{Br}^{\prime}\left(\operatorname{Sym}^{d}(X)\right)
$$

defined in (4.6) is an isomorphism if $d>2 g$. We noted earlier that it is enough to prove the lemma for all $d$ sufficiently large. Therefore, the proof of the lemma is now complete. 


\section{The Cohomology of the Quot Scheme}

For integers $r \geq 1$ and $d$, denote by $\mathcal{Q}(r, d)$ the Quot scheme parameterizing all coherent subsheaves

$$
\mathcal{F} \hookrightarrow \mathcal{O}_{X}^{\oplus r}
$$

where $\mathcal{F}$ is of rank $r$ and degree $-d$. Note that there is no such subsheaf if $d<0$. If $d=0$, then $\mathcal{F}=\mathcal{O}_{X}^{\oplus r}$. If $d=1$, then $\mathcal{Q}(r, d)=X \times \mathbb{C P}^{r-1}$. We assume that $d \geq 1$.

We will now recall from [Bi] a few facts about the Białynicki-Birula decomposition of $\mathcal{Q}(r, d)$. Using the natural action of $\mathbb{G}_{m}=\mathbb{C} \backslash\{0\}$ on $\mathcal{O}_{X}$, we get an action of $\mathbb{G}_{m}^{r}$ on $\mathcal{O}_{X}^{\oplus r}$. This action produces an action of $\mathbb{G}_{m}^{r}$ on $\mathcal{Q}(r, d)$. The fixed points of this torus action correspond to subsheaves of $\mathcal{O}_{X}^{\oplus r}$ that decompose into compatible direct sums

$$
\bigoplus_{i=1}^{r} \mathcal{L}_{i} \hookrightarrow \mathcal{O}_{X}^{\oplus r},
$$

where $\mathcal{L}_{i} \hookrightarrow \mathcal{O}_{X}$ is a subsheaf of rank one. Let $D_{i}$ be the effective divisor given by the inclusion of $\mathcal{L}_{i}$ in $\mathcal{O}_{X}$. In particular, we have $\mathcal{L}_{i}=\mathcal{O}_{X}\left(-D_{i}\right)$.

We use the convention that $\operatorname{Sym}^{0}(X)$ is a single point. Using this notation, we have

$$
\left(D_{1}, \ldots, D_{r}\right) \in \operatorname{Sym}^{m_{1}}(X) \times \cdots \times \operatorname{Sym}^{m_{r}}(X),
$$

where $m_{i}=$ degree $\left(D_{i}\right)$. Conversely, if $\left(D_{1}^{\prime}, \ldots, D_{r}^{\prime}\right) \in \operatorname{Sym}^{m_{1}}(X) \times \cdots \times$ $\operatorname{Sym}^{m_{r}}(X)$, then the point of $\mathcal{Q}(r, d)$ representing the subsheaf

$$
\bigoplus_{i=1}^{r} \mathcal{O}_{X}\left(-D_{i}^{\prime}\right) \subset \mathcal{O}_{X}^{\oplus r}
$$

is fixed by the action of $\mathbb{G}_{m}^{r}$ on $\mathcal{Q}(r, d)$.

For $k \geq 1$, denote by Part $_{r}^{k}$ the set of partitions of $k$ of length $r$. So

$$
\mathbf{m}=\left(m_{1}, \ldots, m_{r}\right) \in \text { Part }_{r}^{k}
$$

if and only if $m_{j}$ are nonnegative integers with

$$
\sum_{j=1}^{r} m_{j}=k \text {. }
$$

For $\mathbf{m} \in$ Part $_{r}^{d}$, define

$$
d_{\mathbf{m}}:=\sum_{i=1}^{r}(i-1) m_{i} .
$$

The connected components of the fixed point locus for the action of $\mathbb{G}_{m}^{r}$ on $\mathcal{Q}(r, d)$ are in bijection with the elements of $\operatorname{Part}_{r}^{d}$. The component corresponding to the partition $\mathbf{m}=\left(m_{1}, \ldots, m_{r}\right)$ is the product

$$
\operatorname{Sym}^{\mathbf{m}}(X):=\operatorname{Sym}^{m_{1}}(X) \times \cdots \times \operatorname{Sym}^{m_{r}}(X) .
$$

It is possible (see [Bi, p. 3]) to choose a one-parameter subgroup $\mathbb{G}_{m} \longrightarrow \mathbb{G}_{m}^{r}$ given by $z \mapsto\left(z^{\lambda_{1}}, z^{\lambda_{2}}, \ldots, z^{\lambda_{r}}\right)$ so that the following two hold: 
(1) The fixed point locus under the induced action of $\mathbb{G}_{m}$ is the same as the fixed point locus under the action of $\mathbb{G}_{m}^{r}$.

(2) The integers $\lambda_{1}<\lambda_{2}<\cdots<\lambda_{r}$ are increasing.

For this action of $\mathbb{G}_{m}$ on $\mathcal{Q}(r, d)$, define

$$
\operatorname{Sym}^{\mathbf{m}}(X)^{+}:=\left\{x \in \mathcal{Q}(r, d) \mid \lim _{t \rightarrow 0} t . x \in \operatorname{Sym}^{\mathbf{m}}(X)\right\},
$$

where $\mathbf{m} \in \mathbf{P a r t}_{r}^{k}$. This stratification of $\mathcal{Q}(r, d)$ gives us a decomposition of the Poincaré polynomial of $\mathcal{Q}(r, d)$. Further, the morphism

$$
\operatorname{Sym}^{\mathbf{m}}(X)^{+} \longrightarrow \operatorname{Sym}^{\mathbf{m}}(X)
$$

that sends a point to its limit is a fiber bundle with fiber $\mathbb{A}^{d_{\mathbf{m}}}$ (see [Bb] and [Bi]), where $d_{\mathbf{m}}$ is defined in (5.1).

This gives

$$
\operatorname{dim} \operatorname{Sym}^{\mathbf{m}}(X)^{+}=\operatorname{dim} \operatorname{Sym}^{\mathbf{m}}(X)+d_{\mathbf{m}}=d+d_{\mathbf{m}}
$$

(see [Bi]).

THEOREM 5.1. For $i \geq 1$,

$$
H^{i}(\mathcal{Q}(r, d), \mathbb{Z}) \cong \bigoplus_{\substack{\mathbf{m} \in \operatorname{Part}_{r}^{d} \\ j+2 d_{\mathbf{m}}=i}} H^{j}\left(\operatorname{Sym}^{m_{1}}(X) \times \cdots \times \operatorname{Sym}^{m_{r}}(X), \mathbb{Z}\right) .
$$

Proof. See [Bi] and [BGL, p. 649, Remark].

We will construct some cohomology classes in $H^{2}(\mathcal{Q}(r, d), \mathbb{Z})$. There is a universal vector bundle $\mathcal{F}^{\text {univ }}$ on $\mathcal{Q}(r, d) \times X$. Fix a point $x_{0} \in X$. Let

$$
i_{x}: \mathcal{Q}(r, d) \longrightarrow \mathcal{Q}(r, d) \times X
$$

be the embedding defined by $z \longmapsto(z, x)$.

Let

$$
c:=i_{x}^{*} c_{1}\left(\mathcal{F}^{\text {univ }}\right) \in H^{2}(\mathcal{Q}(r, d), \mathbb{Z})
$$

be the pullback. This cohomology class $c$ is clearly independent of $x$.

We can produce cohomology classes

$$
\bar{\alpha}_{1}, \bar{\alpha}_{2}, \ldots, \bar{\alpha}_{2 g} \in H^{1}(\mathcal{Q}(r, d), \mathbb{Z})
$$

by taking the slant product of $c_{1}\left(\mathcal{F}^{\text {univ }}\right)$ with the elements of a basis $\left\{\alpha_{1}, \ldots, \alpha_{2 g}\right\}$ for $H^{1}(X, \mathbb{Z})$. Finally, there is a cohomology class $\gamma_{2} \in H^{2}(\mathcal{Q}(r, d), \mathbb{Z})$ obtained by taking the slant product of $c_{2}\left(\mathcal{F}^{\text {univ }}\right)$ with the fundamental class of $X$.

REMARK 5.2. We will see in the next proposition that the cohomology of $\mathcal{Q}(r, d)$ has no torsion. The class $c_{2}\left(\mathcal{F}^{\text {univ }}\right)$ is a $(p, p)$-class and so is the fundamental class of $X$. It follows that the class $\gamma_{2}$ is in the Néron-Severi subgroup of $\mathcal{Q}(r, d)$ since the slant product of two $(p, p)$ classes is in fact $(p, p)$. 
Proposition 5.3. Suppose that $d \geq 2$. Then the classes

$$
c, \gamma_{2}, \bar{\alpha}_{i} \cup \bar{\alpha}_{j}, \quad 1 \leq i<j \leq 2 g,
$$

generate $H^{2}(\mathcal{Q}(r, d), \mathbb{Z})$. In fact, $H^{2}(\mathcal{Q}(r, d), \mathbb{Z})$ is torsionfree, and these classes form a basis of the $\mathbb{Z}$-module $H^{2}(\mathcal{Q}(r, d), \mathbb{Z})$.

Proof. Using Theorem 5.1 and Theorem 3.1, it follows that $H^{2}(\mathcal{Q}(r, d), \mathbb{Z})$ is torsionfree of rank

$$
\left(\begin{array}{c}
2 g \\
2
\end{array}\right)+2 .
$$

Hence, it suffices to show the stated classes generate the second cohomology group.

The torus action on $\mathcal{Q}(r, d)$ induces a Białynicki-Birula stratification on this variety, as described before. Using (5.3), the cell of largest dimension in the Białynicki-Birula decomposition is the cell corresponding to the partition

$$
\mathbf{m}_{1}=(0,0,0, \ldots, d),
$$

and the second largest cell corresponds to the partition

$$
\mathbf{m}_{2}=(0,0, \ldots, 0,1, d-1) .
$$

It follows that $\operatorname{Sym}^{\mathbf{m}_{1}}(X)^{+}$is an open dense subscheme of $\mathcal{Q}(r, d)$. Let $D:=$ $\mathcal{Q}(r, d) \backslash \operatorname{Sym}^{\mathbf{m}_{1}}(X)^{+}$be the complement. Using (5.2), we have

$$
H^{2}\left(\operatorname{Sym}^{\mathbf{m}_{1}}(X)^{+}, \mathbb{Z}\right)=H^{2}\left(\operatorname{Sym}^{\mathbf{m}_{1}}(X), \mathbb{Z}\right) .
$$

Further, by a dimension calculation (5.3) and a Gysin sequence,

$$
H^{0}(D, \mathbb{Z}) \cong H^{0}\left(\operatorname{Sym}^{\mathbf{m}_{2}}(X), \mathbb{Z}\right) .
$$

Let

$$
\iota: \operatorname{Sym}^{\mathbf{m}_{1}}(X) \hookrightarrow \mathcal{Q}(r, d)
$$

be the inclusion map.

The Gysin sequence for the decomposition $\mathcal{Q}(r, d)=\operatorname{Sym}^{\mathbf{m}_{1}}(X)^{+} \bigsqcup D$ now reads:

$$
\begin{aligned}
& \cdots \longrightarrow \longrightarrow H^{0}\left(\operatorname{Sym}^{\mathbf{m}_{2}}(X), \mathbb{Z}\right) \stackrel{f_{*}}{\longrightarrow} H^{2}(\mathcal{Q}(r, d), \mathbb{Z}) \\
& \stackrel{\iota^{*}}{\longrightarrow} H^{2}\left(\operatorname{Sym}^{\mathbf{m}_{1}}(X), \mathbb{Z}\right) \longrightarrow \cdots,
\end{aligned}
$$

where

$$
f: \operatorname{Sym}^{d-1}(X) \times X \longrightarrow \mathcal{Q}(r, d)
$$

is the embedding. From [CS], this sequence splits, or in other words, the Białynicki-Birula stratification is integrally perfect.

To complete the proof, it suffices to verify the following two statements:

(S1) The classes $\iota^{*}\left(\bar{\alpha}_{i} \cup \bar{\alpha}_{j}\right), \quad 1 \leq i<j \leq 2 g$, and $\iota^{*}(c)$ generate $H^{2}\left(\operatorname{Sym}^{\mathbf{m}_{1}}(X), \mathbb{Z}\right)$.

(S2) The class $\gamma_{2}$ generates the image of $f_{*}$. 
For (S1), observe that

$$
\iota^{*}\left(\mathcal{F}^{\text {univ }}\right)=j_{z}^{*} \mathcal{O}_{\operatorname{Sym}^{d}(X) \times X}\left(-D^{\text {univ }}\right) \oplus \mathcal{O}_{\operatorname{Sym}^{d}(X)}^{r-1},
$$

where $j_{z}: \operatorname{Sym}^{d}(X) \longrightarrow \operatorname{Sym}^{d}(X) \times X$ is the embedding defined by $z \longmapsto(z, x)$, and $D^{\text {univ }}$ is the universal divisor on $\operatorname{Sym}^{d}(X) \times X$. From Proposition 3.2 and Theorem 3.1 it follows that the classes

$$
\iota^{*}(c), \quad \iota^{*}\left(\bar{\alpha}_{i} \cup \bar{\alpha}_{j}\right), \quad 1 \leq i<j \leq 2 g,
$$

give a basis for $H^{2}\left(\operatorname{Sym}^{\mathbf{m}_{1}}(X), \mathbb{Z}\right)$. Further, $\gamma_{2} \in \operatorname{kernel}\left(\iota^{*}\right)$.

For (S2), we assume that $r=2$ for simplicity. The proof in the case of higher rank is obtained by adding some trivial summands to the argument below.

As noted before, we have a split exact sequence:

$$
\begin{aligned}
0 \longrightarrow & H^{0}\left(\operatorname{Sym}^{d-1}(X) \times X, \mathbb{Z}\right) \stackrel{f_{*}}{\longrightarrow} H^{2}(\mathcal{Q}(r, d), \mathbb{Z}) \\
& \stackrel{i^{*}}{\longrightarrow} H^{2}\left(\operatorname{Sym}^{d}(X), \mathbb{Z}\right) \longrightarrow 0 .
\end{aligned}
$$

Fix some quotient

$$
q: \mathcal{O}_{X} \longrightarrow Q \longrightarrow 0
$$

of degree $d-1$ and also fix some quotient

$$
q^{\prime}: \mathcal{O}_{X} \longrightarrow \mathcal{O}_{p} \longrightarrow 0
$$

of degree 1 , where $p \in X$ is a point not in the support of $Q$.

This gives us a point $z \in \operatorname{Sym}^{d-1}(X) \times X$. We can expand this to a morphism

$$
F: \mathbb{A}^{1} \longrightarrow\left(\operatorname{Sym}^{d-1}(X) \times X\right)^{+}
$$

by considering the family of quotients

$$
F(t):=\left(\begin{array}{cc}
q f & 0 \\
t q^{\prime} & q^{\prime}
\end{array}\right): \mathcal{O}_{X}^{\oplus 2} \longrightarrow Q \oplus \mathcal{O}_{p} \longrightarrow 0 .
$$

Taking the closure of $F\left(\mathbb{A}^{1}\right)$ in $\mathcal{Q}(2, d)$, we obtain an inclusion

$$
F: \mathbb{P}^{1} \hookrightarrow \mathcal{Q}(2, d) .
$$

Since $\operatorname{dim} \mathcal{Q}(2, d)=2 d$, this gives a cohomology class

$$
\left[\mathbb{P}^{1}\right] \in H^{4 d-2}(\mathcal{Q}(2, d), \mathbb{Z}) .
$$

Let

$$
\mathcal{W} \longrightarrow \mathbb{P}^{1} \times X
$$

be the restriction of the universal vector bundle $\mathcal{F}^{\text {univ }} \longrightarrow \mathcal{Q}(2, d) \times X$. It fits in the short exact sequence

$$
0 \longrightarrow \mathcal{W} \longrightarrow \mathcal{O}_{\mathbb{P}^{1} \times X}^{\oplus 2} \longrightarrow \widetilde{Q}:=\left(\mathcal{O}_{\mathbb{P}^{1}} \otimes Q\right) \oplus\left(\mathcal{O}_{\mathbb{P}^{1}}(1) \otimes \mathcal{O}_{p}\right) \longrightarrow 0 .
$$

Note that the Chern character

$$
\operatorname{Ch}(\widetilde{Q})=d \omega_{X}+\omega_{X} \cup \omega_{\mathbb{P}^{1}},
$$


where $\omega_{X}$ and $\omega_{\mathbb{P}^{1}}$ are the fundamental classes of $X$ and $\mathbb{P}^{1}$, respectively. In particular, $c_{1}(\widetilde{Q})=\omega_{X}$. Therefore, the slant product of $c_{1}(\widetilde{Q})$ with elements of $H^{1}(X, \mathbb{Z})$ vanish. We have

$$
c_{2}(\widetilde{Q})=\omega_{X} \cup \omega_{\mathbb{P}^{1}} .
$$

Its slant product with $X$ is then just $\omega_{\mathbb{P}^{1}}$. Therefore,

$$
\left(F^{*} \gamma_{2}\right) \cup\left[\mathbb{P}^{1}\right]=\int_{\mathbb{P}^{1}} \gamma_{2}=1 .
$$

So the cohomology classes described in the statement of the proposition give a basis for the vector space $H^{2}(\mathcal{Q}(r, d), \mathbb{Q})$.

We will prove the following statements:

$$
\begin{gathered}
\left(F^{*} c\right) \cup\left[\mathbb{P}^{1}\right]=0, \\
\alpha_{i} \cup\left[\mathbb{P}^{1}\right]=0, \\
f_{*}\left(\left[\operatorname{Sym}^{d-1}(X) \times X\right]\right) \cup\left[\mathbb{P}^{1}\right]=1, \\
\left(F^{*} \gamma_{2}\right) \cup\left[\mathbb{P}^{1}\right]=\int_{\mathbb{P}^{1}} \gamma_{2}=1 .
\end{gathered}
$$

The map $f$ is defined in (5.5).

We first show that these statements complete the proof. For that, it is sufficient to observe that they imply that both

$$
f_{*}\left(\left[\operatorname{Sym}^{d-1}(X) \times X\right]\right) \text { and } \gamma_{2}
$$

are dual to $\left[\mathbb{P}^{1}\right]$ and hence must be equal.

To prove (5.8), consider (5.6). Choose a point $x \in X$ away from the support of $Q \oplus \mathcal{O}_{p}$ and restrict $\mathcal{W}$ to $\mathbb{P}^{1} \times\{x\}$. From (5.7) it follows that the first Chern class of this restriction vanishes. The first Chern class of this restriction clearly coincides with $\left(F^{*} c\right) \cup\left[\mathbb{P}^{1}\right]$.

The left-hand side of (5.9) is clearly the slant product of $c_{1}(\mathcal{W})$ with $\alpha_{i}$. We noted before that this slant product vanishes.

Now, (5.10) is clear from the construction of the morphism $F$ from $\mathbb{P}^{1}$. Finally, (5.11) has already been proved.

\section{The Cohomological Brauer Group}

For integers $r \geq 1$ and $d \geq 0$, take any subsheaf $\mathcal{F} \subset \mathcal{O}_{X}^{\oplus r}$ lying in $\mathcal{Q}(r, d)$. Taking the $r$ th exterior power, we get a subsheaf $\bigwedge^{r} \mathcal{F} \subset \wedge^{r} \mathcal{O}_{X}^{\oplus r}=\mathcal{O}_{X}$. Let

$$
\varphi: \mathcal{Q}(r, d) \longrightarrow \operatorname{Sym}^{d}(X)
$$

be the morphism that sends any subsheaf $\mathcal{F} \subset \mathcal{O}_{X}^{\oplus r}$ to the scheme theoretic support of the quotient $\mathcal{O}_{X} / \bigwedge^{r} \mathcal{F}$. Let

$$
\varphi^{*}: \operatorname{Br}^{\prime}\left(\operatorname{Sym}^{d}(X)\right) \longrightarrow \operatorname{Br}^{\prime}(\mathcal{Q}(r, d))
$$

be the pullback homomorphism using $\varphi$.

Lemma 6.1. The homomorphism $\varphi^{*}$ in (6.2) is an isomorphism. 
Proof. Note that $\operatorname{Br}^{\prime}(\mathcal{Q}(r, d))=\operatorname{Br}^{\prime}\left(\operatorname{Sym}^{d}(X)\right)=0$ if $d \leq 1$. Therefore, we assume that $d \geq 2$.

The cohomology group $H^{3}(\mathcal{Q}(r, d), \mathbb{Z})$ is torsionfree. Indeed, this follows from Theorem 5.1 and the fact that $H^{*}\left(\operatorname{Sym}^{n}(X), \mathbb{Z}\right)$ is torsionfree [Ma, p. 329, (12.3)]. Therefore, Proposition 2.1 says that

$$
\operatorname{Br}^{\prime}(\mathcal{Q}(r, d))=\left(H^{2}(\mathcal{Q}(r, d), \mathbb{Z}) / \mathrm{NS}(\mathcal{Q}(r, d))\right) \otimes_{\mathbb{Z}}(\mathbb{Q} / \mathbb{Z}) .
$$

Let

$$
\varphi^{\prime}: H^{2}\left(\operatorname{Sym}^{d}(X), \mathbb{Z}\right) \longrightarrow H^{2}(\mathcal{Q}(r, d), \mathbb{Z})
$$

be the pullback homomorphism using $\varphi$ in (6.1). Recall from Theorem 3.1 the description of $H^{2}\left(\operatorname{Sym}^{d}(X), \mathbb{Z}\right)$. From Proposition 5.3 we conclude that $\varphi^{\prime}$ is injective, and

$$
H^{2}(\mathcal{Q}(r, d), \mathbb{Z})=\operatorname{image}\left(\varphi^{\prime}\right) \oplus \mathbb{Z} \cdot \gamma_{2},
$$

where $\gamma_{2}$ is the cohomology class in Proposition 5.3. Take any point

$$
y:=\left(y_{1}, \ldots, y_{d}\right) \in \operatorname{Sym}^{d}(X)
$$

such that all $y_{i}$ are distinct. Then $\varphi^{-1}(y)$ is a product of copies of $\mathbb{C P}^{r-1}$, and hence

$$
H^{1}\left(\varphi^{-1}(y), \mathbb{Z}\right)=0 .
$$

From this it follows that the image of the cup product

$$
H^{1}(\mathcal{Q}(r, d), \mathbb{Z}) \otimes H^{1}(\mathcal{Q}(r, d), \mathbb{Z}) \longrightarrow H^{2}(\mathcal{Q}(r, d), \mathbb{Z})
$$

is in the image $\varphi^{\prime}$. If the point $x \in X$ in (5.4) is different from all $y_{i}$, then the restriction of the universal vector bundle $\mathcal{F}^{\text {univ }}$ (see $\left.(5.4)\right)$ to $\varphi^{-1}(y)$ is the trivial vector bundle of rank $r$. From this it follows that $c$ is in the image of $\varphi^{\prime}$.

From (6.4) it follows immediately that

$$
\mathrm{NS}(\mathcal{Q}(r, d))=\varphi^{\prime}\left(\operatorname{NS}\left(\operatorname{Sym}^{d}(X)\right)\right) \oplus \mathbb{Z} \cdot \gamma_{2} .
$$

In view of (6.3), from this we conclude that $\varphi^{*}$ in (6.2) is an isomorphism if $d \geq 2$.

As before, fix a point $x_{0} \in X$. Let

$$
\delta: \mathcal{Q}(r, d) \longrightarrow \mathcal{Q}(r, d+r)
$$

be the morphism that sends any $\mathcal{F} \subset \mathcal{O}_{X}^{\oplus r}$ represented by a point of $\mathcal{Q}(r, d)$ to the point representing the subsheaf $\mathcal{F} \otimes \mathcal{O}_{X}\left(-x_{0}\right) \subset \mathcal{O}_{X}^{\oplus r}$. Let

$$
\delta^{*}: \operatorname{Br}^{\prime}(\mathcal{Q}(r, d+r)) \longrightarrow \operatorname{Br}^{\prime}(\mathcal{Q}(r, d))
$$

be the pullback homomorphism by $\delta$.

COROllary 6.2. For any $d \geq 2$, the homomorphism $\delta^{*}$ in (6.6) is an isomorphism. 
Proof. As in (6.1), define

$$
\psi: \mathcal{Q}(r, d+r) \longrightarrow \operatorname{Sym}^{d+r}(X)
$$

to be the morphism that sends any subsheaf $\mathcal{F} \subset \mathcal{O}_{X}^{\oplus r}$ to the scheme theoretic support of the corresponding quotient $\left(\bigwedge^{r} \mathcal{O}_{X}^{\oplus r}\right) /\left(\bigwedge^{r} \mathcal{F}\right)$. Let

$$
h: \operatorname{Sym}^{d}(X) \longrightarrow \operatorname{Sym}^{d+r}(X)
$$

be the morphism defined by $\sum_{x \in X} n_{x} \cdot x \longmapsto r \cdot x_{0}+\sum_{x \in X} n_{x} \cdot x$. The diagram of morphisms

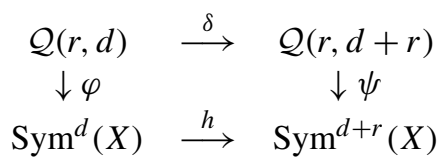

is commutative, where $\varphi$ and $\delta$ are defined in (6.1) and (6.5), respectively. Consider the corresponding commutative diagram

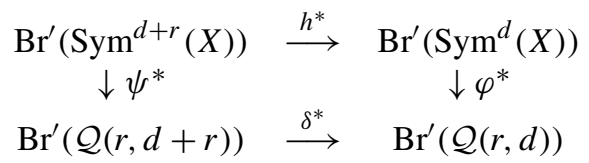

of homomorphisms. If $d \geq 2$, from Lemma 6.1 we know that $\psi^{*}$ and $\varphi^{*}$ are isomorphisms, whereas Lemma 4.1 implies that $h^{*}$ is an isomorphism. Therefore, the homomorphism $\delta^{*}$ is an isomorphism.

REMARK 6.3. We are grateful to an unknown referee for this comment. We give here an alternative proof of the fact that the pullback map $\varphi^{*}$ induces an isomorphism on cohomology. Consider the big cell of the Białynicki-Birula decomposition described before. It corresponds to the partition

$$
\mathbf{m}_{1}=(0,0,0, \ldots, d) .
$$

We have a Zariski locally trivial fibration

$$
\rho: \operatorname{Sym}^{\mathbf{m}_{1}}(X)^{+} \longrightarrow \operatorname{Sym}^{\mathbf{m}_{1}}(X)
$$

with fiber $\mathbb{A}^{n}$, see $[B B$, p. 492]. We claim that we have an induced isomorphism

$$
\operatorname{Br}^{\prime}\left(\operatorname{Sym}^{\mathbf{m}_{1}}(X)^{+}\right) \cong \operatorname{Br}^{\prime}\left(\operatorname{Sym}^{\mathbf{m}_{1}}(X)\right) .
$$

To see this, we will use the exact sequence in Proposition 2.1, which is valid for noncompact spaces; see [Sco, p. 878]. The morphism $\rho$ induces an isomorphism in cohomology groups since it has contractible fibers. Although the morphism $\rho$ may not be a vector bundle, the Néron-Severi groups of the two varieties agree under the identification of cohomology groups as before; see [Fu, p. 22, Proposition 1.9]. It follows now from Proposition 2.1 and Lemma 5 that $\rho^{*}$ induces an isomorphism on cohomological Brauer groups.

The morphism $\varphi: \operatorname{Sym}^{\mathbf{m}_{1}}(X) \longrightarrow \operatorname{Sym}^{d}(X)$ is an isomorphism. So we have a diagram 


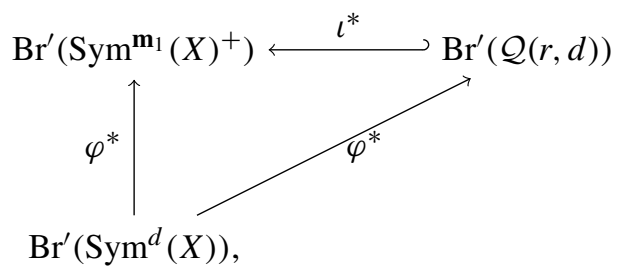

$\iota$ is the composition $\operatorname{Sym}^{\mathbf{m}_{1}}(X)^{+} \hookrightarrow \operatorname{Sym}^{\mathbf{m}_{1}}(X) \longrightarrow \mathcal{Q}(r, d)$; we note that the homomorphism $\varphi^{*}$ in the left is an isomorphism. The map $i^{*}$ is injective by [Mi, IV, Corollary 2.6]. We can now deduce that $\varphi^{*}$ is an isomorphism.

ACKNOWLEDGMENTS. We thank the two referees for detailed and helpful comments. Remark 6.3 is due to a referee. The first-named author is supported by a J. C. Bose Fellowship.

\section{References}

[ACGH] E. Arbarello, M. Cornalba, P. A. Griffiths, and J. Harris, Geometry of algebraic curves, Vol. I, Grundlehren Math. Wiss., 267, Springer-Verlag, New York, 1985.

[BBGN] V. Balaji, I. Biswas, O. Gabber, and D. S. Nagaraj, Brauer obstruction for a universal vector bundle, C. R. Math. Acad. Sci. Paris 345 (2007), 265-268.

[Ba] J. M. Baptista, On the $L^{2}$-metric of vortex moduli spaces, Nuclear Phys. B 844 (2011), 308-333.

[BB] A. Białynicki-Birula, Some theorems on actions of algebraic groups, Ann. of Math. (2) 98 (1973), 480-497.

[BDW] A. Bertram, G. Daskalopoulos, and R. Wentworth, Gromov invariants for holomorphic maps from Riemann surfaces to Grassmannians, J. Amer. Math. Soc. 9 (1996), 529-571.

[Bb] A. Białynicki-Birula, Some theorems on actions of algebraic groups, Ann. of Math. (2) 98 (1973), 480-497.

[Bi] E. Bifet, Sur les points fixes schéma Quot $_{\mathcal{O}_{X} / X, k}$ sous l'action du tore $\mathbf{G}_{m, k}^{r}$, C. R. Math. Acad. Sci. Paris 309 (1989), 609-612.

[BGL] E. Bifet, F. Ghione, and M. Letizia, On the Abel-Jacobi map for divisors of higher rank on a curve, Math. Ann. 299 (1994), 641-672.

[BH] I. Biswas and Y. I. Holla, Brauer group of moduli of principal bundles over a curve, J. Reine Agnew. Math. 677 (2013), 225-249.

[BR] I. Biswas and N. M. Romão, Moduli of vortices and Grassmann manifolds, Comm. Math. Phys. 320 (2013), 1-20.

[CS] J. Carrel and A. Sommese, Some topological aspects of $\mathbb{C}^{*}$ actions on compact Kähler manifolds, Comment. Math. Helv. 54 (1979), 567-582.

[dJ] A. J. de Jong, A result of Gabber, 〈http://www.math.columbia.edu/ dejong/ papers/2-gabber.pdf).

[Dh] A. Dhillon, On the cohomology of moduli of vector bundles and the Tamagawa number of $\mathrm{SL}_{n}$, Canad. J. Math. 54 (2006), 1000-1025.

[Fu] W. Fulton, Intersection theory, Second edition, Ergeb. Math. Grenzgeb. (3), 2, Springer-Verlag, Berlin, 1998.

[Ga] O. Gabber, Some theorems on Azumaya algebras, The Brauer group, Lecture Notes in Math., 844, pp. 129-209, Springer, Berlin-New York, 1981. 
[Ma] I. G. Macdonald, Symmetric products of an algebraic curve, Topology 1 (1962), 319-343.

[Mi] J. S. Milne, Étale cohomology, Princeton Math. Ser., 33, Princeton University Press, Princeton, NJ, 1980.

[Ne] F. Neumann, Algebraic stacks and moduli of vector bundles, Publicações Matemáticas do IMPA, Colóquio Brasileiro de Matemática, 27, 2009.

[Sco] S. Schröer, Topological methods for complex-analytic Brauer groups, Topology 44 (2005), 875-894.

[Scb] R. L. E. Schwarzenberger, Jacobians and symmetric products, Illinois J. Math. 7 (1963), 257-268.

I. Biswas

School of Mathematics

Tata Institute of Fundamental

Research

Homi Bhabha Road

Bombay 400005

India

\section{indranil@math.tifr.res.in}

J. Hurtubise

Department of Mathematics

McGill University

Burnside Hall

805 Sherbrooke St. W.

Montreal, QC H3A 0B9

Canada

\author{
A. Dhillon \\ Department of Mathematics \\ Middlesex College \\ University of Western Ontario \\ London, ON N6A 5B7 \\ Canada
}

adhill3@uwo.ca

jacques.hurtubise@mcgill.ca 